\title{
OSCILLATION CRITERIA FOR SECOND ORDER NONLINEAR DIFFERENTIAL EQUATIONS WITH INTEGRABLE COEFFICIENTS
}

\author{
JAMES S. W. WONG
}

(Communicated by Kenneth R. Meyer)

\begin{abstract}
Consider the second order nonlinear differential equation $y^{\prime \prime}+$ $a(t) f(y)=0$ where $a(t) \in C[0, \infty), f(y) \in C^{1}(-\infty, \infty), f^{\prime}(y) \geq 0$, and $y f(y)>0$ for $y \neq 0$. Furthermore, $f(y)$ also satisfies either a superlinear or a sublinear condition, which covers the prototype nonlinear function $f(y)=|y|^{\gamma}$ sgn $y$ with $\gamma>1$ and $0<\gamma<1$ respectively. The coefficient $a(t)$ is allowed to be negative for arbitrarily large values of $t$ and is integrable in the sense that the improper interval $\int_{t}^{\infty} a(s) d s=A(t)$ exists for each $t \geq 0$. Oscillation criteria involving integrals of $A(t)$ due to Coles and Butler for the superlinear and sublinear cases are shown to remain valid without the additional hypothesis that $A(t) \geq 0$.
\end{abstract}

We consider the second order nonlinear differential equation

$$
y^{\prime \prime}+a(t) f(y)=0, \quad t \in[0, \infty),
$$

where $a(t) \in C[0, \infty)$ and $f(y) \in C^{1}(-\infty, \infty)$, and $f^{\prime}(y) \geq 0$ for all $y$ and satisfies $y f(y)>0$ if $y \neq 0$. The prototype of equation (1) is the so-called Emden-Fowler equation

$$
y^{\prime \prime}+a(t)|y|^{\gamma} \operatorname{sgn} y=0, \quad \gamma>0 .
$$

Here we are interested in the oscillation of solutions of (1) when $f(y)$ also satisfies the following superlinear condition:

$$
0<\int_{\varepsilon}^{\infty} \frac{d y}{f(y)}, \quad \int_{-\infty}^{-\varepsilon} \frac{d y}{f(y)}<\infty, \quad \text { for all } \varepsilon>0,
$$

which corresponds to the special case $f(y)=|y|^{\gamma} \operatorname{sgn} y$ when $\gamma>1$, and the following sublinear condition:

$$
0<\int_{0}^{\varepsilon}, \quad \int_{-\varepsilon}^{0} \frac{d y}{f(y)}<\infty, \quad \text { for all } \varepsilon>0,
$$

which corresponds to the special case $f(y)=|y|^{\gamma} \operatorname{sgn} y$ when $0<\gamma<1$. These assumptions were first introduced systematically in [17]. The coefficient $a(t)$

Received by the editors September 21, 1990 and, in revised form, December 7, 1990.

1980 Mathematics Subject Classification (1985 Revision). Primary 34C10, 34C15.

Key words and phrases. Second order, nonlinear, ordinary differential equations, oscillation, asymptotic behavior. 
is allowed to be negative for arbitrarily large values of $t$, and in addition, we assume that its integral over the nonnegative reals is finite. In other words, the improper integral $A(t)=\int_{t}^{\infty} a(s) d s$ exists and is finite for each $t \geq 0$. Under these circumstances, in general not every solution to the second order nonlinear differential equation (1) is continuable throughout the entire half real axis. For this reason, we confine ourselves with those solutions of (1) that exist and can be continued on some interval of the form $\left[t_{0}, \infty\right)$ where $t_{0} \geq 0$ may depend on the particular solution. Such a solution is said to be oscillatory if it has arbitrarily large zeros. Equation (1) is called oscillatory if all continuable solutions are oscillatory.

In the superlinear case, the first oscillation theorem for equation (2) is that of Atkinson [1] who proved if $a(t) \geq 0$ then

$$
\lim _{T \rightarrow \infty} \int_{0}^{T} t a(t) d t=\infty
$$

is sufficient for oscillation of (2) when $\gamma>1$. This result was improved by Kiguradze [10] by removing the requirement that $a(t) \geq 0$. On the other hand, Coles [7] showed that if $A(t) \geq 0$ then (5) can be further relaxed to

$$
\lim _{T \rightarrow \infty} \int_{0}^{T} A(t) d t=\infty .
$$

In the sublinear case, the first oscillation result for equation (2) is due to Belohorec [2] who proved that if $a(t) \geq 0$ then

$$
\lim _{T \rightarrow \infty} \int_{0}^{T} t^{\gamma} a(t) d t=\infty
$$

is sufficient for oscillation of (2) when $0<\gamma<1$. In a subsequent paper, Belohorec [3] showed that the condition $a(t) \geq 0$ was superfluous, thus providing the analogue to Kiguradze's result in the superlinear case. When $A(t) \geq 0$ Butler [5] proved the analogue of Coles's result; namely, the condition that

$$
\lim _{T \rightarrow \infty} \int_{0}^{T} t^{\gamma-1} A(t) d t=\infty
$$

is sufficient for oscillation of (2) when $0<\gamma<1$. If $A(t) \geq 0$ then (7) implies (8); so Butler's result extends the original theorem of Belohorec. When $A(t)$ exists for each $t \geq 0$, equation (1) is often referred to as having integrable coefficients. There is extensive literature on oscillation of (1) in this special situation. In the linear case, i.e., $f(y)=y$, we refer to [16] and in the nonlinear case, that of Butler [4].

In this paper, we consider the natural question: can the conditions $A(t) \geq 0$ in the oscillation theorems of Coles [7] and Butler [5] be removed? In a recent article [19], we improved Coles's result by showing that $A(t) \geq 0$ is indeed superfluous. Here we will prove that the same is true for Butler's theorem. It will be shown that our arguments are in fact sufficient to obtain extensions of Coles's and Butler's results to the more general equation (1). More specifically, we prove the following superlinear extension.

Theorem 1. Let $f(y)$ satisfy the superlinearity condition (3). Also let $A(t)$ exist for each $t \geq 0$ and satisfy condition (6). Then equation (1) is oscillatory. 
The sublinear case is more difficult and will require additional assumptions of the nonlinear function in the following condition:

$$
f^{\prime}(y) F(y) \geq \frac{1}{c} \text { for all } y,
$$

where $c>0$ and $F(y)=\int_{0}^{y} d v / f(v)$. In the special case $f(y)=|y|^{\gamma} \operatorname{sgn} y$, $c=(1-\gamma) / \gamma$. Condition (9) has been found useful in other oscillation theorems (see Philos [15] and also [18, 20]). This assumption was first introduced by Coles [8] in the following form:

$$
\frac{F^{\prime \prime}(y) F(y)}{F^{\prime 2}(y)} \leq-\frac{1}{c} \quad \text { for all } y .
$$

Subsequent applications indicated that (9) is in fact more convenient to use than its equivalent form above. Thus, we shall prove

Theorem 2. Let $f(y)$ satisfy the sublinearity condition (4) and also (9). Suppose $A(t)$ exists for each $t \geq 0$ and satisfies

$$
\lim _{T \rightarrow \infty} \int_{0}^{T} t^{\lambda-1} A(t) d t=\infty,
$$

where $\lambda=1 /(c+1)<1$. Then equation (1) is oscillatory.

Crucial to the proofs of the above two theorems is the following result due to Kwong and Wong [11]:

Proposition 1. Let $A(t)$ exist for each $t \geq 0$. Suppose $y(t)$ is a nonoscillatory solution of $(1)$ on $\left[t_{0}, \infty\right)$. Then the integral equation

$$
\frac{y^{\prime}(t)}{f(y(t))}=\alpha+A(t)+\int_{t}^{\infty} \frac{f^{\prime}(y(s))\left(y^{\prime}(s)\right)^{2}}{f^{2}(y(s))} d s
$$

is satisfied for $t \geq t_{0}$, where $\alpha$ is a nonnegative constant. Moreover, if the condition

$$
\lim _{y \rightarrow \pm \infty} f(y)= \pm \infty
$$

is satisfied then $\alpha=0$.

Proof of Theorem 1. Since $f(y)$ satisfies (3), it clearly satisfies (12); hence the integral equation (11) is satisfied with $\alpha=0$. Let $y(t)$ be a nonoscillatory solution of equation (1), and it satisfies the integral equation (11) with $\alpha=0$. Dropping the last term in (11), we have the inequality

$$
\frac{y^{\prime}(t)}{f(y(t))} \geq A(t) \text {. }
$$

Integrating (13) from $t_{0}$ to $t$ and denoting $G(y)=\int_{y}^{\infty} d v / f(v)$, we find

$$
\int_{t_{0}}^{t} \frac{y^{\prime}(s)}{f(y(s))} d s=\int_{y\left(t_{0}\right)}^{y(t)} \frac{d v}{f(v)}=G\left(y\left(t_{0}\right)\right)-G(y(t)) ;
$$

hence

$$
G\left(y\left(t_{0}\right)\right)-G(y(t)) \geq \int_{t_{0}}^{t} A(s) d s \rightarrow \infty
$$

as $t \rightarrow \infty$. This is the desired contradiction, so equation (1) is oscillatory. 
Proof of Theorem 2. We begin by noting that if $f(y)$ satisfies (9) then (12) is also satisfied. In fact, from (9) and the definition of $F(y)$, we find $F^{\prime}(y)=$ $1 / f(y)$ and

$$
\frac{f^{\prime}(y)}{f(y)} \geq \frac{1}{c} \frac{F^{\prime}(y)}{F(y)} .
$$

Integrating above from $y_{0}$ to $y$, one obtains

$$
\log \frac{f(y)}{f\left(y_{0}\right)} \geq \frac{1}{c} \log \frac{F(y)}{F\left(y_{0}\right)}
$$

hence

$$
\left(\frac{f(y)}{f\left(y_{0}\right)}\right)^{c} \geq \frac{F(y)}{F\left(y_{0}\right)} .
$$

Applying (9) again to the inequality in the above line, we note

$$
c f^{\prime}(y)(f(y))^{c} \geq \frac{1}{F(y)}(f(y))^{c} \geq \frac{f\left(y_{0}\right)^{c}}{F\left(y_{0}\right)} .
$$

Integrating once more from $y_{0}$ to $y$ yields

$$
\left(\frac{c}{c+1}\right)\left(f(y)^{c+1}-f\left(y_{0}\right)^{c+1}\right) \geq \frac{f\left(y_{0}\right)^{c}}{F\left(y_{0}\right)}\left(y-y_{0}\right),
$$

which shows that $f(y) \rightarrow+\infty$ as $y \rightarrow \infty$. A similar argument applies for $y<y_{0}$ and results in $f(y) \rightarrow-\infty$ as $y \rightarrow-\infty$, proving (12). So if $y(t)$ is any nonoscillatory solution of (1), then it satisfies the integral equation (11) with $\alpha=0$. In particular, we have

$$
\int_{t_{0}}^{\infty} f^{\prime}(y) \frac{y^{\prime 2}(s)}{f(y(s))^{2}} d s<\infty
$$

from which we shall show that $y(t)$ has the following asymptotic behavior:

$$
\lim _{t \rightarrow \infty} \frac{F(y(t))}{t}=0 \text {. }
$$

To prove (15), we apply the Schwarz Inequality and estimate $F(y(t))$ as follows:

$$
\begin{aligned}
F(y(t))-F\left(y\left(t_{1}\right)\right) & =\left|\int_{t_{1}}^{t} \frac{y^{\prime}(s)}{f(y(s))} d s\right| \\
& \leq\left(\int_{t_{1}}^{t} \frac{f^{\prime}(y) y^{\prime 2}}{f(y)^{2}} d s\right)^{1 / 2}\left(\int_{t_{1}}^{t} \frac{1}{f^{\prime}(y)} d s\right)^{1 / 2},
\end{aligned}
$$

where $t_{1} \geq t_{0}$. In view of (14), we choose $t_{1}$ sufficiently large so that for any $\varepsilon>0$,

$$
\int_{t_{1}}^{\infty} \frac{f^{\prime}(y) y^{\prime 2}}{f(y)^{2}} d s<\frac{\varepsilon}{4}
$$

Using (17) and condition (9) in (16), one obtains

$$
F(y(t)) \leq F\left(y\left(t_{1}\right)\right)+\frac{\sqrt{c \varepsilon}}{2}\left(\int_{t_{1}}^{t} F(y(s)) d s\right)^{1 / 2} .
$$


Suppose $F(y(t)) \in L^{1}\left(t_{1}, \infty\right)$. Then $F(y(t))$ is bounded by (18) so (15) is satisfied. Otherwise, we can choose $t_{2} \geq t_{1}$ so that

$$
F\left(y\left(t_{1}\right)\right) \leq \frac{\sqrt{c \varepsilon}}{2}\left(\int_{t_{1}}^{t} F(y) d s\right)^{1 / 2} \quad \text { for } t \geq t_{2}
$$

which together with (18), yields

$$
F(y(t)) \leq \sqrt{c \varepsilon}\left(\int_{t_{1}}^{t} F(y(s)) d s\right)^{1 / 2}
$$

Upon integrating (19), we have for $t \geq t_{2}$,

$$
\left.\left(\int_{t_{1}}^{t} F(y) d s\right)\right)^{1 / 2}-\left(\int_{t_{1}}^{t_{2}} F(y) d s\right)^{1 / 2} \leq \frac{\sqrt{c \varepsilon}}{2}\left(t-t_{2}\right) \leq \frac{\sqrt{c \varepsilon}}{2} t .
$$

Once again we can choose $t_{3}$ so that $\int_{t_{1}}^{t_{2}} F(y) d s<c \varepsilon t^{2} / 4$ for all $t \geq t_{3}$. With (19) and (20), this proves the desired assertion (15).

We define the function $\phi(t)=t^{\lambda-1} F(y(t))$ and note by differentiation that

$$
\frac{y^{\prime}(t)}{f(y(t))}=t^{1-\lambda} \phi^{\prime}(t)+(1-\lambda) t^{-\lambda} \phi(t)
$$

which combines with condition (9) to give

$$
f^{\prime}(y) \frac{y^{\prime 2}}{f(y)^{2}} \geq \frac{1}{c} t^{1-\lambda} \frac{\phi^{\prime 2}}{\phi}+2 \lambda t^{-\lambda} \phi^{\prime}+\lambda(1-\lambda) t^{-1-\lambda} \phi .
$$

We further note that integrating the second term of the right-hand side of (21) yields for $T \geq t$,

$$
2 \lambda \int_{t}^{T} s^{-\lambda} \phi^{\prime}(s) d s=2 \lambda\left\{\frac{\phi(T)}{T^{\lambda}}-\frac{\phi(t)}{t^{\lambda}}+\lambda \int_{t}^{T} \frac{\phi(s)}{s^{\lambda+1}} d s\right\} .
$$

On account of (15) and the fact that $\phi(t)$ is nonnegative, the limits of the two integrals in (22) both exist and could be infinity. Noting (14) and $\lambda<1$, we can integrate both sides of inequality (21) and deduce that the limit of the integral on the left-hand side of (22) must be finite. Hence the integral on the right-hand side of (22) is likewise finite, and therefore, we can define an energy function

$$
\Sigma(t)=\phi(t)-(1+\lambda) t^{\lambda} \int_{t}^{\infty} \frac{\phi(s)}{s^{\lambda+1}} d s .
$$

We note that $y(t)$ is a solution of equation (1) and also satisfies the integral equation (11) with $\alpha=0$. Computing $\Sigma^{\prime}(t)$, we find

$$
\begin{aligned}
\Sigma^{\prime}(t)= & t^{\lambda-1} \cdot \frac{y^{\prime}(t)}{f(y(t))}+(\lambda-1) t^{\lambda-2} F(y(t)) \\
& -\lambda(1+\lambda) t^{\lambda-1} \int_{t}^{\infty} \frac{\phi(s)}{s^{\lambda+1}} d s+(1+\lambda) t^{\lambda} \frac{\phi(t)}{t^{\lambda+1}} .
\end{aligned}
$$

Combining the second and last terms in the above and using (11), one obtains

$$
\begin{aligned}
\Sigma^{\prime}(t)= & t^{\lambda-1}\left(A(t)+\int_{t}^{\infty} \frac{f^{\prime}(y) y^{\prime 2}}{f(y)^{2}} d s\right)+2 \lambda \frac{\phi(t)}{t} \\
& -\lambda(1+\lambda) t^{\lambda-1} \int_{t}^{\infty} \frac{\phi(s)}{s^{\lambda+1}} d s,
\end{aligned}
$$


where $\lambda=1 /(1+c)<1, c=(1-\lambda) / \lambda>0$. Furthermore, we apply (15) and combine (21) and (22) in (24) to obtain

$$
\Sigma^{\prime}(t) \geq t^{\lambda-1} A(t)+\frac{1}{c} t^{\lambda-1} \int_{t}^{\infty} s^{1-\lambda} \frac{\phi^{\prime 2}(s)}{\phi(s)} d s .
$$

Since the integral term above is nonnegative, we have $\Sigma^{\prime}(t) \geq t^{\lambda-1} A(t)$, which implies that $\Sigma(t) \rightarrow \infty$ as $t \rightarrow \infty$ in view of (8). From the definition of $\Sigma(t)$ in (23), we also deduce that $\phi(t) \rightarrow \infty$ as $t \rightarrow \infty$. Define $t_{n}=\sup \{t: \phi(t) \leq n\}$, which satisfies $t_{n} \rightarrow \infty$ as $n \rightarrow \infty$, and also $t \geq t_{n}$ implies $\phi(t) \geq n=\phi\left(t_{n}\right)$. Substituting $t$ in (23) by $t_{n}$ and using its definition, we obtain

$$
\begin{aligned}
\Sigma\left(t_{n}\right) & =\phi\left(t_{n}\right)-(1+\lambda) t_{n}^{\lambda} \int_{t_{n}}^{\infty} \frac{\phi(s)}{s^{\lambda+1}} d s \\
& \geq \phi\left(t_{n}\right)-(1+\lambda) t_{n}^{\lambda} \phi\left(t_{n}\right) \int_{t_{n}}^{\infty} \frac{d s}{s^{\lambda+1}} \\
& =\phi\left(t_{n}\right)\left\{1-(1+\lambda) \frac{1}{\lambda}\right\}=-\frac{1}{\lambda} \phi\left(t_{n}\right)<0,
\end{aligned}
$$

which contradicts the fact that $\Sigma\left(t_{n}\right) \rightarrow \infty$ as $n \rightarrow \infty$. The proof is now complete.

Remark 1. When $f(y)=|y|^{\gamma} \operatorname{sgn} y, c=(1-\gamma) / \gamma$ and $\lambda=\gamma$, so condition (10) becomes (8).

Remark 2. We note that the theorem of Kwong and Wong [11] has been used by others, notably by Naito [14] in proving results on asymptotic behavior of nonoscillatory solutions of equation (1) (see also [13]).

Remark 3. In Coffman and Wong [6], we advocated the theory of "duality principle" for superlinear and sublinear equations in that every oscillation and nonoscillation theorem for the superlinear equation has its natural analogue for the sublinear equation and vice versa. In most cases known to us, by setting $\gamma=1$ in an oscillation criterion, one often obtains a new oscillation result. Here we may refer to conditions (5) and (7) and also their extensions (6) and (8). See also Kwong and Wong [12, 13], and Erbe [9].

Remark 4. It is easy to give examples of $a(t)$ such that (6) is satisfied by not (5). Consider

$$
A(t)=\frac{1}{t}+\frac{2 \sin t}{\sqrt{t}}
$$

and so

$$
a(t)=\frac{1}{t^{2}}+\frac{\sin t}{t^{3 / 2}}-\frac{2 \cos t}{\sqrt{t}} .
$$

Here $A(t)$ is not nonnegative and the improper integral $\int^{\infty} s a(s) d s$ does not exist. Hence, neither Coles's [7] nor Kiguradze's [10] theorem would apply. Similarly, for the sublinear equation (2), $0<\gamma<1$, we can also take

$$
A(t)=\frac{1}{t^{\gamma}}+\frac{\sqrt{t} \sin t}{t^{\gamma}}
$$

Here

$$
a(t)=\frac{\gamma}{t^{\gamma+1}}+\frac{\gamma \sqrt{t} \sin t}{t^{\gamma+1}}+\frac{\sqrt{t} \cos t}{t^{\gamma}}
$$


fails to satisfy Belohorec's condition (7), but $A(t)$ assumes negative values for large values of $t$ and satisfies (8); so by Theorem 2 we have oscillation of equation (2).

Remark 5. Finally, we give nontrivial examples of nonlinear functions satisfying conditions (3), (4), and (9). In the superlinear case, the example $f(y)=e^{y}-$ 1 clearly satisfies (3). The function $f(y)=y\left(|y|^{-1 / 2}+|y|^{1 / 2}\right)$ satisfies both conditions (3) and (4). Moreover, it satisfies condition (9), since in this instance, $F(y)=2 \tan ^{-1} \sqrt{y}$ and condition (9) is satisfied with $c=1$.

\section{REFERENCES}

1. F. V. Atkinson, On second order nonlinear oscillation, Pacific J. Math. 5 (1955), 643-647.

2. S. Belohorec, Oscillatory solutions of certain nonlinear differential equations of second order, Mat. Fyz. Casopis Sloven Akad Vied. 11 (1961), 250-255.

3. _ Two remarks on the properties of solutions of a nonlinear differential equation, Acta Tac. Rerum. Natur. Univ. Comen. Math. 22 (1969), 19-26.

4. G. J. Butler, On the oscillatory behavior of a second order nonlinear differential equation, Ann. Mat. Pura Appl. (4) 105 (1975), 73-92.

5. __ An integral criterion for the oscillation of a second order sublinear ordinary differential equation, Indian J. Math, 24 (1982), 1-7.

6. C. V. Coffman and J. S. W. Wong, Oscillation and nonoscillation theorems for second order ordinary differential equations, Funkcial. Ekvac. 15 (1972), 119-130.

7. W. J. Coles, Oscillation criteria for nonlinear second order equations, Ann. Mat. Pura Appl. (4) 82 (1969), 123-134.

8. A A nonlinear oscillation theorem, International Conference on Differential Equations (H. A. Antosiewicz, ed.), Academic Press, New York, 1975, pp. 193-202.

9. L. H. Erbe, Nonoscillation criteria for second order nonlinear differential equations, J. Math. Anal. Appl. 108 (1985), 515-527.

10. I. T. Kiguradze, A note on the oscillation of solution of the equation $u^{\prime \prime}+a(t)|u|^{n} \operatorname{sgn} u=0$, Casopis Pest. Mat. 92 (1967), 343-350.

11. M. K. Kwong and J. S. W. Wong, An application of integral inequality to second order nonlinear oscillation, J. Differential Equations 46 (1982), 63-77.

12. _ Nonoscillation theorems for a second order sublinear ordinary differential equation, Proc. Amer. Math. Soc. 87 (1983), 467-474.

13. Linearization of second order nonlinear oscillation theorems, Trans. Amer. Math. Soc. 279 (1983), 705-722.

14. M. Naito, Asymptotic behavior of solutions of second order differential equations with integrable coefficients, Trans. Amer. Math. Soc. 282 (19884), 577-588.

15. Ch. G. Philos, Oscillation criteria for second order superlinear differential equations, Canad. J. Math. 41 (1989), 321-340.

16. J. S. W. Wong, Oscillation and nonoscillation of solutions of second order linear differential equations with integrable coefficients, Trans. Amer. Math. Soc. 144 (1969), 197-215.

17. __ Oscillation theorems for second order nonlinear differential equations, Bull. Inst. Math. Acad. Sincia 3 (1975), 283-309.

18. __ A sublinear oscillation theorem, J. Math. Anal. Appl. 139 (1989), 408-412.

19. __ Oscillation theorems for second order nonlinear differential equations, Proc. Amer. Math. Soc. 106 (1989), 1069-1077.

20. $\ldots$, Oscillation of sublinear second order differential equations with integral coefficients, $\mathrm{J}$. Math. Anal. Appl. (to appear). 